\title{
Auf die Couch! Beziehungsprobleme zwischen Rational Choice und Politischer Psychologie
}

\author{
Johannes Marx/Christine Tiefensee
}

\section{Einleitung}

In Diskussionen um die Politische Psychologie stößt man immer wieder auf die Meinung, dass die empirische Politische Psychologie und Rational Choice-Theorien in einem direkten Konkurrenzverhältnis zueinander stünden (Hoggett u. Thompson 2012, S. 1; Lodge u. Taber 2013, S. xiii; implizit etwa auch Green u. Shapiro 1996). Rational Choice-Ansätze konstruierten Akteure als rational kalkulierende Entscheider, wohingegen die Politische Psychologie betone, dass Entscheidungsprozesse maßgeblich durch Faktoren wie Heuristiken, Emotionen, Persönlichkeitsmerkmale und Lernmechanismen beeinflusst seien. Während Rational Choice Präferenzordnungen von Akteuren innerhalb theoretischer Modelle axiomatisch setze, sei Politische Psychologie an der empirischen Erhebung tatsächlicher Einstellungen interessiert. Wo Rational Choice eine homogene Wirkung von Strukturen auf Akteure postuliere, stelle die Politische Psychologie klar, dass aufgrund unterschiedlicher Persönlichkeitsmerkmale keine solche einheitliche Wirkung angenommen werden könne. Und während Rational Choice die Einfachheit und den weiten Anwendungsbereich ihrer Modelle preise, rühmten sich Politische Psychologen, die Black Box der Entscheidungsfindung zu öffnen und somit eine empirisch adäquatere, und damit bessere Erklärung von Entscheidungsprozessen zu liefern.

In diesem Beitrag möchten wir diesem angeblichen Konkurrenzverhältnis zwischen Politischer Psychologie und Rational Choice auf den Grund gehen. Anhand einer systematischen Untersuchung der jeweiligen wissenschaftstheoretischen Voraussetzungen beider Forschungsprogramme werden wir zeigen, dass der Unterschied zwischen Rational Choice und Politischer Psychologie trotz aller Verschiedenheiten nicht so groß ist, wie zunächst angenommen. Vielmehr werden wir argumentieren, dass auch die Politische Psychologie immer von einer minimalen Rationalitätsunterstellung ausgehen muss, während nicht nur realistisch gedeutete, sondern selbst instrumentell-strukturalistisch interpretierte Rational ChoiceTheorien implizit auf psychologische Wirkmechanismen angewiesen sind.

Wir werden diese These in drei Schritten entwickeln. Im Rahmen unserer ersten Teilthese werden wir zunächst zeigen, weswegen Politische Psychologie nicht ohne Rationalitätsannahmen, wie sie auch von Rational Choice-Theorien vertreten werden, auskommen kann. In einem zweiten Schritt werden wir uns Rational ChoiceTheorien, und damit unserer zweiten Teilthese, zuwenden und zwischen einer realistischen und einer instrumentalistischen Interpretation von Rational Choice unterscheiden. Unser Ziel wird sein, zu argumentieren, dass die realistische Inter- 
pretation von Rational Choice mit dem Ansatz der Politischen Psychologie zusammenfällt, dass aber auch instrumentalistische Rational Choice-Theorien, die zu erklärende Varianzen komplett auf strukturelle Änderungen zurückzuführen suchen, ebenfalls psychologisch gedeutet werden müssen. Ergebnis unserer Diskussion wird dementsprechend sein, dass kein striktes Konkurrenzverhältnis zwischen Politischer Psychologie und Rational Choice besteht. Dennoch stellt sich in Anbetracht fortbestehender Unterschiede die Frage, zu welchem Grad Erklärungen psychologisch fundiert oder eher Rational Choice basiert sein sollten. In einem letzten Schritt werden wir eine erste Antwort auf diese Frage vorschlagen. Ein kurzes Fazit fasst unsere Argumentation abschließend zusammen.

Doch bevor wir in unsere Argumentation einsteigen, sind zwei Vorbemerkungen angebracht. Um Missverständnissen vorzubeugen, möchten wir erstens darauf hinweisen, dass wir uns darüber bewusst sind, dass wir die beiden Erklärungsprogramme auf einer sehr abstrakten Ebene diskutieren. Von einer Politischen Psychologie zu reden, wäre hierbei jedoch genauso irreführend, wie von einer Rational Choice-Theorie auszugehen. Politische Psychologie und Rational Choice sind vielmehr als übergreifende Bezeichnungen zu verstehen, unter die eine Vielzahl verschiedener Annahmen und Theorien zusammengefasst werden. Wie in den nächsten Abschnitten klar werden wird, zeichnen sich die jeweiligen Theorien und Annahmen dennoch durch Merkmale aus, die es ermöglichen, Politische Psychologie und Rational Choice als separate Forschungsprogramme bzw. -ansätze zu behandeln. Zweitens muss klargestellt werden, welche Bedingungen erfüllt sein müssen, um überhaupt sinnvoll von einem Konkurrenzverhältnis zwischen Rational Choice und Politischer Psychologie reden zu können. Wir schlagen folgende Bedingungen vor:

(A) Forschungsgegenstand. Politische Psychologie und Rational Choice müssen denselben Forschungsgegenstand untersuchen. Politisch-psychologische und Rational Choice-basierte Erklärungen können nur dann miteinander konkurrieren und verglichen werden, wenn es beide zum Ziel haben, dasselbe Explanandum zu erklären.

(B) Erklärungsbegriff. Die Erklärungen beider Ansätze müssen an denselben Qualitätsmaßstäben gemessen werden können: Ohne einen solchen geteilten Maßstab ließen sich keine vergleichenden Aussagen über die alternativen Erklärungen treffen.

Im Folgenden werden wir nur Fälle betrachten, in denen $(A)$ gegeben ist. Dies hat gewisse Einschränkungen zur Folge. Zum einen impliziert es, dass Rational Choice wie die Politische Psychologie als eine deskriptive Theorie und nicht wie von manchen vorgeschlagen als normative Theorie verstanden werden muss. Würde sie als normative Theorie interpretiert, die analysiert, wie Akteure rational handeln sollten, wohingegen Politische Psychologie untersucht, wie sich Akteure tatsächlich verhalten, wäre kein gemeinsamer Forschungsgegenstand und damit auch keine Konkurrenzgrundlage gegeben. Zum anderen bedingt die Erfüllung von $(A)$ einen Fokus auf Erklärungen sozialer Makrophänomene unter Verweis auf individuelles Handeln. Da das Forschungsinteresse von Rational Choice im 
Gegensatz zur Politischen Psychologie nicht darauf abzielt, beispielsweise die Entstehung von Persönlichkeitsmerkmalen zu erklären, konkurriert sie in dieser Hinsicht auch nicht mit Politischer Psychologie. Es wäre jedoch vorschnell, diesen Unterschied bezüglich des Forschungsgegenstands von Rational Choice und Politischer Psychologie lediglich als einen Fall unterschiedlicher Forschungsinteressen abzutun. Politische Psychologen interessieren sich nicht einfach für die Entstehung von Einstellungen, wohingegen Rational Choice-Theoretiker dieses Interesse nun einmal nicht teilen. Vielmehr gehen Politische Psychologen davon aus, dass eine Erklärung von Makrophänomenen und individuellem Handeln nur dann adäquat ist, wenn auch die der Handlung unterliegenden Faktoren erklärt sind. Anders ausgedrückt: Wie wir in den nächsten Abschnitten näher ausführen werden, sind Unterschiede hinsichtlich des Forschungsgegenstands der Politischen Psychologie und von Rational Choice auf die unterschiedlichen Gütekriterien zurückzuführen, die Politische Psychologen und Rational Choice-Theoretiker an sozialwissenschaftliche Erklärungen anlegen. Dennoch teilen beide in Einklang mit $(B)$ den fundamentalen Anspruch, Kausalerklärungen für die jeweiligen Explananda auf Basis des methodologischen Individualismus zu entwickeln.

Zusätzlich zu Bedingungen $(A)$ und $(B)$, ohne deren Erfüllung keine Konkurrenz vorliegen könnte, stellen wir zwei weitere Bedingungen auf, die das Ausmaß des angeblichen Konkurrenzverhältnisses betreffen:

(C) Umfang. Um umfassende Konkurrenz herzustellen, muss der politisch-psychologische Ansatz mit allen Interpretationen des Rational Choice-Ansatzes - vor allem mit realistischen und instrumentalistischen Deutungen - in Konflikt stehen: Andernfalls würden nur manche Rational Choice-Vertreter die Argumente Politischer Psychologen ablehnen, wohingegen andere diese akzeptieren könnten.

(D) Exklusivität. Die konkurrierenden Erklärungen müssen sich gegenseitig ausschließen: Es darf nicht der Fall sein, dass sich eine der Erklärungen als Spezialfall in die andere Erklärung integrieren lässt.

Wie in der Hauptthese unseres Beitrags behauptet, sind Bedingungen $(C)$ wie auch $(D)$ verletzt. Dies zu zeigen, ist Ziel der nächsten beiden Abschnitte.

\section{Der rationale Kern der Politischen Psychologie}

Wie bereits angedeutet, vereinigt der politisch-psychologische Ansatz viele verschiedene Theorien und Forschungsansätze, die von einem Fokus auf Persönlichkeitsmerkmale und Charakterzüge über verschiedene Informationsverarbeitungsabläufe bis hin zu neuronalen Prozessen führen. Wir werden uns im Folgenden auf die empirisch orientierten Theorien der Politischen Psychologie konzentrieren, die sich erstens an dem Anspruch messen lassen, Handlungen eines Akteurs im begrifflichen Vokabular psychischer Variablen kausal zu erklären, und die zweitens dem methodologischen Individualismus verpflichtet sind. 


\subsection{Politisch-psychologische Erklärungen}

Politische Psychologie gehört insofern zur Psychologie, als sie sich für die psychischen Merkmale handelnder Akteure und damit die psychologischen Aspekte, die Handlungen und Einstellungen beeinflussen und steuern, interessiert. Sie ist politisch, da sie sich mit Einstellungen und Handlungen in dezidiert politischen Kontexten befasst. ${ }^{1}$ Demnach lässt sich der Forschungsgegenstand der Politischen Psychologie sowohl auf der Makro- als auch der Mikroebene verorten. Auf der Makroebene zielt sie auf die Erklärung sozialer Phänomene ab, wie z.B. auf Wahlen, die Bildung öffentlicher Meinung und kollektives Verhalten. Im Einklang mit dem methodologischen Individualismus erfolgt diese Erklärung unter Bezug auf die Mikroebene, und damit auf individuelles Verhalten. Dementsprechend geht der Erklärungsanspruch der Politischen Psychologie erstens über den der klassischen Psychologie hinaus, die primär an der Erklärung und Interpretation von lediglich individuellem Handeln interessiert ist. Zweitens verfolgt sie dabei auch ein umfassenderes Forschungsgebiet als Rational Choice, da sie nicht nur die Erklärung von intentionalen Handlungen als einer Teilklasse menschlichen Verhaltens, sondern auch die Erklärung von Verhalten generell, z.B. im Sinne unbewusster psychologischer Prozesse, zum Ziel hat.

Insgesamt ist kennzeichnend für Theorien der Politischen Psychologie, dass für die Erklärung sozialer Phänomene der Rekurs nicht nur auf individuelles Handeln, sondern auf die Ebene der Persönlichkeit der Akteure als notwendig erachtet wird. Der Grund hierfür liegt darin, dass empirischen Befunden zufolge Individuen über unterschiedliche Modi der Informationsverarbeitung und Entscheidungsfindung verfügen und diese mit persönlichen Merkmalen der Akteure in Verbindung stehen (Capara et al. 1999). Dies führt innerhalb der Politischen Psychologie bei der Rekonstruktion der individuellen Situationswahrnehmung und Handlungsselektion zu einer besonderen Betonung individueller Faktoren, wie sie sich in den folgenden Merkmalen widerspiegelt:

(a) Deskriptiver Anspruch auf empirische Adäquatheit. Politische Psychologen vertreten den Anspruch, empirisch adäquate Erklärungen für individuelles Handeln und Verhalten zu entwickeln. Aus Perspektive der Politischen Psychologie zielt eine sozialwissenschaftliche Erklärung somit auf die Identifikation der tatsächlichen Ursachen sozialer Phänomene und auf die empirisch adäquate Abbildung individueller Entscheidungsprozesse ab (Farnham 1990). Demnach wird die Frage nach den spezifischen Variablen, die diese Prozesse beeinflussen, als empirisch offen betrachtet. Dies führt zu einer Öffnung der Black Box von Entscheidungsprozessen in zweierlei Hinsicht.

1 Wir folgen damit dem in der Politischen Psychologie gängigen Verständnis, das Wort ,politisch nicht im Sinne einer substantiell politischen Positionierung zu verstehen - die politische Psychologie verfolgt als solche kein politisches Ziel - sondern lediglich als Spezifizierung des Anwendungsbereichs dieser empirischen Forschungsrichtung. Dies liegt in unserer Zielsetzung begründet, da wir uns für die Erklärungskraft der ausgewählten Theorien interessieren. Für einen Überblick über die historische Entwicklung und den Forschungsgegenstand der Politischen Psychologie, siehe u.a. Catellani 2004, Schoen 2006, Monroe et al. 2009 und Marcus 2013. 
(b) Öffnung der Black Box: Einstellungen und Informationsverarbeitung. Theorien der Politischen Psychologie stimmen mit Rational Choice-Theorien überein in der Betonung der Relevanz von Überzeugungen (beliefs) und Präferenzen (preferences) für die Handlungserklärung. Da allerdings in zahlreichen experimentellen Studien systematische Verletzungen der von Rational Choice gemeinhin postulierten Rationalitätsansprüche an Präferenzordnungen und Belief-Sets nachgewiesen wurden, rücken die kognitive Struktur der Akteure und ihre individuelle Weise der Informationsverarbeitung in den Fokus der Politischen Psychologie (Kahneman u. Tversky 1981; Kahneman et al. 1991; Tversky 1975; Fazio 1990). Hierbei werden weder Rationalitätsanforderungen an die akteursspezifischen Belief-Sets noch an die der Präferenzen gestellt. Politisch-psychologische Theorien lassen somit Raum für Fehlwahrnehmungen, die Rolle von Heuristiken, inkonsistente Überzeugungen, intransitive Präferenzordnungen, Lerneffekte sowie den Wandel von Überzeugungen und Präferenzen im Zeitverlauf. Sollte dies für empirische Adäquatheit nötig sein, kann die Ebene von Einstellungen verlassen werden, um auch neuronale Prozesse und genetische Faktoren in Erklärungen zu integrieren (Marcus 2013).

(c) Öffnung der Black Box: Handlungsselektion. Auch der Prozess der Handlungsselektion wird innerhalb der Politischen Psychologie in besonderer Weise betrachtet. Während Rational Choice-Theorien den Aspekt der Nutzenmaximierung als zentrale Gesetzmäßigkeit der Handlungsselektion betonen, halten psychologische Theorien diesen Aspekt prinzipiell offen und bieten theoretische Erklärungen nicht nur für rationale, nutzenmaximierende Handlungswahlen, sondern auch für weitere Modi der Handlungsselektion. Eine besondere Rolle spielen hierbei Emotionen. Emotionen sind für Informationsverarbeitung wie auch die Entscheidungsfindung relevant, da sie sowohl bei der Speicherung von Informationen wie auch bei der Aktivierung von Einstellungen kausal wirksam sind: Emotionen beeinflussen Kognition, Motivation und Handeln eines Akteurs in vielfältiger Weise (Rothermund und Eder 2011; Small et al. 2006). Obwohl emotionale Prozesse oft zunächst unterhalb der Schwelle der bewussten kognitiven Kontrolle stattfinden, haben sie dennoch Einfluss auf das Handeln der Akteure.

(d) Stopp-Bedingungen. Wenn auch eine politisch-psychologische Erklärung klassischerweise im Vokabular von Einstellungen formuliert ist, findet sich innerhalb der Politischen Psychologie keine Stopp-Bedingung derart, dass Erklärungen von Akteursaktivität lediglich auf die Ebene von Überzeugungen und Präferenzen rekurrieren sollten. Stattdessen werden Einstellungen, die zu Handlungen führen, erstens nicht als gegeben angenommen, sondern selbst als erklärungsbedürftig betrachtet, so dass Einstellungen für die Politische Psychologie nicht exogene Faktoren, sondern Teil ihres Forschungsgegenstands sind. Zweitens werden Einstellungen hinsichtlich ihrer Funktion noch weiter ausdifferenziert und in neueren Strömungen der Politischen Psychologie unter Bezug auf neuronale Prozesse erweitert. Politisch-psychologische Erklärungen stoppen daher nicht unbedingt auf der Ebene der Einstellungen, sondern können auch neuronale Prozesse oder genetische Faktoren heranziehen. Wie tief eine Erklärung vordringen muss, ist folglich innerhalb der Politischen Psychologie prinzipiell offen. 


\title{
2.2 Die Notwendigkeit von Rationalitätsunterstellungen in der Politischen Psychologie
}

Diese kurzen Erläuterungen mögen den Eindruck erwecken, dass alle Annahmen innerhalb der Politischen Psychologie offen für empirische Untersuchung sind: $\mathrm{Ob}$ Fragen wie ,Maximieren Akteure Erwartungsnutzen?‘, ,Sind ihre Präferenzen konsistent?‘, ,Schätzen sie Wahrscheinlichkeiten kohärent ein?‘ oder ,Sind Akteure rational?‘ zu bejahen oder zu verneinen sind, müsse empirische Forschung zeigen. Doch dieser Schein trügt. Wie wir nun zeigen werden, muss auch die Politische Psychologie davon ausgehen, dass Akteure zumindest minimalen Rationalitätsstandards genügen.

Der Grund hierfür liegt in den Zuschreibungskriterien für handlungswirksame Einstellungen, also Präferenzen und Überzeugungen. Wie Hempel (1961, S. 1318) ausführt, entsprächen diese Einstellungen nicht lediglich Reiz-Reaktions-Mechanismen, sondern seien als weitgefasste Dispositionen des Akteurs zu verstehen. Betrachte man nun aber, wie diese Dispositionen spezifiziert werden, so sehe man, dass Überzeugungen und Präferenzen in Bezug aufeinander individuiert werden. In Hempels Worten (1961, S. 16):

\begin{abstract}
The statement that Henry wants a drink of water implies, among other things, that Henry is disposed to drink a liquid offered him - provided that he believes it to be potable water (and provided he has no overriding reasons for refusing to accept it, et cetera). Thus, ascription of an objective here has implications concerning characteristic overt behavior only when taken in conjunction with ascriptions of appropriate beliefs. Similarly, (...) the hypothesis that Henry believes the streets to be slushy implies the occurrence of characteristics [sic] overt behavior only when taken in conjunction with suitable hypotheses about Henry's objectives.

And indeed it seems that generally a hypothesis about an agent's objectives can be taken to imply the occurrence of specific overt action only when conjoined with appropriate hypotheses about his beliefs; and vice versa.
\end{abstract}

Überzeugungs- und Präferenzzuschreibungen sind demnach epistemisch interdependent. Wie ist diese epistemische Interdependenz nun zu erklären? Weswegen implizieren Aussagen über die Ziele eines Akteurs Handlungen nur in Konjunktion mit Überzeugungen? Die Antwort findet sich in folgendem Prinzip $R$ :

$(R)$ Ein Akteur mit dem Ziel $z$ führt diejenige Handlung $h$ aus, die vor dem Hintergrund seiner eigenen Überzeugungen $\ddot{u}$ der Wahl adäquater Mittel zur Erreichung von $z$ entspricht.

Dieses Prinzip begründet, weswegen Aussagen über Henrys Wunsch nach Wasser (z) Henrys Disposition implizieren, die angebotene Flüssigkeit zu trinken (b), solange er glaubt, dass diese Flüssigkeit Wasser ist $(\ddot{u})$. Gleichermaßen implizieren Aussagen über Henrys Überzeugungen zum Zustand der Straßen ( $\ddot{u})$ nur dann, dass er disponiert ist, lieber zu Fuß zu gehen als das Auto zu nehmen ( $h$ ), wenn er beabsichtigt, sicher unterwegs zu sein $(z)$. Und letztlich werten wir Henrys Verhalten nur dann als Handlung $(b)$, wenn es sich auf seine Ziele $(z)$ und Überzeugun- 
gen $(\ddot{i})$ zurückführen lässt. Anders ausgedrückt, wird das Dreieck aus Zielen $z$, Überzeugungen $\ddot{u}$ und Handlungen $h$ erst geformt durch Prinzip $R$ : Es ist dieses Prinzip, welches die epistemische Interdependenz von Einstellungszuschreibungen erklärt. Prinzip $R$ ist nun allerdings nichts anderes, als das Rationalitätsprinzip: Es umfasst die klassische Definition instrumenteller Rationalität, welche Rationalität an die Wahl adäquater Mittel zur Zielerreichung knüpft. ${ }^{2}$

Für die Politische Psychologie bedeutet das nun Folgendes: Wenn wir Akteuren Überzeugungen und Ziele aufgrund ihrer Handlungen zuschreiben, setzen wir damit ihre Rationalität immer schon voraus. ${ }^{3}$ Denn die Überprüfung wie auch die Begründung von bestimmten Einstellungszuschreibungen unterstellt immer schon, dass der Akteur rational ist, dass er also diejenige Handlungsoption wählt, die vor dem Hintergrund seiner Überzeugungen für seine Zielerreichung am erfolgversprechendsten ist. Wie Hempel (1961, S. 17) zusammenfasst, impliziert dies „that the way in which we use a person's actions as evidence in ascertaining his goals has the presupposition of rationality built into it. " Ließen sich die Dispositionen eines Akteurs nicht in dieses rationale Dreieck von Zielen, Überzeugungen und Handlungen einbetten - hätte ein Individuum Dispositionen, die sich nicht durch das Prinzip $R$ strukturieren ließen - könnten wir weder von Zielen und Überzeugungen, noch von einem intentional handelnden Akteur reden. Folglich sind es keine offenen Fragen, ob Akteure rational oder ihre Einstellungen konsistent sind. Insofern die Politische Psychologie auf Einstellungen handelnder, intentionaler Akteure rekurriert, kann sie nicht umhin, Akteuren Rationalität zuzusprechen. ${ }^{4}$

Heißt das, die Politische Psychologie ist überflüssig, so dass wir uns ganz und gar auf Rational Choice konzentrieren sollten? Zwei Einwände legen nahe, dass dieser Schluss verfrüht wäre. Erstens können Politische Psychologen auf empirische Studien verweisen, die belegen, dass Akteure Rationalitätsannahmen oft verletzen - die Studien von Kahneman und Tversky seien hier nochmals stellvertretend erwähnt (Kahneman u. Tversky 1981; Kahneman et al. 1991; Tversky 1975). Dieser Verweis auf empirische Studien ist unserer Meinung nach absolut legitim. Allerdings müssen daraus gezogene Schlüsse mit Sorgfalt betrachtet werden. Denn was diese empirischen Studien zeigen, ist nicht, dass die Rationalität von Akteuren grundsätzlich bezweifelt werden kann, sondern dass es eine offene

2 Dieses Prinzip darf nicht mit Formeln beispielsweise der SEU-Theorie verwechselt werden, die eine mathematische Repräsentation dieses Prinzips darstellen. Vgl. unsere Anmerkungen hierzu in Kapitel 3.1.

3 Dies wirft Fragen zum Status des Rationalitätsprinzips auf. So interpretiert Davidson 1984, 2004 Prinzip $R$ als normatives Prinzip, das konstitutiv für Interpretationen ist. Hempel 1961 hingegen hält an einem empirischen Status fest. Unser Beitrag muss die Diskussion um den Status von Prinzip $R$ ausklammern. Für eine Analyse des Einflusses dieser Diskussion auf die Realismus/ Instrumentalismus-Debatte um Rational Choice-Theorien, siehe Tiefensee (i.E.).

4 An dieser Stelle könnte eingewandt werden, dass dieses Ergebnis wenig ergiebig sei, da es auf einem völlig entleerten Rationalitätsbegriff beruht. Da eben dieses Rationalitätsverständnis allerdings genau jenes ist, das Rational Choice-Theorien zugrunde liegt, kann dieser Punkt keine Kritik an einer Studie sein, die das angebliche Konkurrenzverhältnis von Politischer Psychologie und Rational Choice-Theorien untersucht. Außerdem ist es unserer Meinung nach von großem Interesse, dass ein Rationalitätsbegriff, der eben keine substantielle Anforderungen an die Inhalte von Präferenzen und Überzeugungen richtet, eine solche zentrale Rolle in der Zuschreibung propositionaler Einstellungen spielt. Vgl. auch Fußnote 3. 
Frage ist, zu welchem Grad Akteure rational sind. Obwohl einem Individuum nur dann Ziele und Überzeugungen zugesprochen werden können, wenn sein Verhalten grundsätzlich vor dem Hintergrund des Rationalitätsprinzips interpretierbar ist, heißt dies nicht, dass seine Einstellungen vollkommen konsistent oder seine Handlungen immer rational sein müssen. Lokalisierte Formen der Irrationalität sowie systematische Abweichungen von Rationalitätsstandards, wie sie von der Politischen Psychologie aufgedeckt werden, sind mit der zentralen Rolle des Rationalitätsprinzips im Rahmen von Einstellungszuschreibungen kompatibel. Politisch-psychologische Untersuchungen, die das Ziel verfolgen, die Black Box der Einstellungen und der Entscheidungsfindung zu öffnen, verlieren folglich trotz der Zentralität von Rationalitätsannahmen nicht ihre Relevanz, greifen gleichzeitig aber die Annahme minimaler Rationalität nicht an. ${ }^{5}$

Zweitens beschäftigt sich die Politische Psychologie wie bereits oben erwähnt nicht nur mit Handlungen und Einstellungen, sondern auch allgemeiner mit Verhalten und neuronalen oder gar genetischen Faktoren, die Verhalten beeinflussen. Da sich unser Argument zum Rationalitätsprinzip aber lediglich auf Einstellungen und Handlungen bezieht, können Politische Psychologen folglich erwidern, dass dieses Untersuchungen zu allgemeinerem Verhalten und neuronalen Prozessen völlig unangetastet lasse. Diese Beobachtung stimmt sicherlich; allerdings muss auch sie mit Vorsicht behandelt werden. Sie ist insofern richtig und wichtig, als menschliches Handeln und Verhalten nicht zwangsläufig in der Sprache von Einstellungen beschrieben werden muss, sondern auch in rein naturwissenschaftlichem Vokabular wie dem der Neurobiologie gefasst werden kann. Die Beobachtung wäre jedoch irreführend, wenn sie implizieren wollte, dass politischpsychologische Erklärungen den Rekurs auf Einstellungen komplett hinter sich lassen und rein naturwissenschaftliches Vokabular verwenden könnten. Dies ist darin begründet, dass einstellungsbasierte Erklärungen nicht nur die Ursachen einer Handlung explizieren, sondern auch die Handlungsgründe eines Akteurs. ${ }^{6}$ Handlungsgründe bestehen nach Davidson (1980a) aus belief/desire-Paaren, welche die Handlungsmotive des Akteurs explizieren, wobei Handlungsursachen in physikalischen, z.B. neuronalen Prozessen zu verorten sind. Es ist nun genau dieser Doppelcharakter intentionaler Erklärungen, der uns die Handlungen eines Akteurs im Sinne seiner Handlungsgründe sowohl verstehen als auch im Sinne von Handlungsursachen erklären lässt, welcher die Attraktivität des intentionalen $\mathrm{Zu}-$

5 Folglich sind psychologisch orientierte Theorien, wie zum Beispiel die Prospect Theory, nicht als vollkommen neue, eigenständige Theorien zu verstehen, sondern als Erweiterungen und Modifikationen von rationalitätsbasierten Theorien, um systematische Abweichungen von Rationalitätsstandards integrieren zu können.

6 Zum diffizilen Verhältnis zwischen mentaler und physikalischer Sprache sowie zu Einstellungen und neuronalen Prozessen, siehe Davidson 1980b, 2004. Zum Verhältnis zwischen Gründen und Ursachen siehe Davidson 1980a. Davidson argumentiert dort, dass eine (Token-)Identitätsbeziehung zwischen mentalen Zuständen einerseits und physikalischen Zuständen andererseits besteht, wodurch sowohl mithilfe mentaler Sprache als auch physikalischer Sprache auf dasselbe kausal wirksame Ereignis referiert werden kann, obwohl mentale Sprache nicht auf physikalische Sprache reduzierbar ist. Der Verweis auf einen Handlungsgrund erklärt wiederum nur dann eine Handlung, wenn diese durch diesen Handlungsgrund verursacht wurde. Für eine ausführliche Betrachtung dieser Problematik und ihrer Relevanz für die Sozialwissenschaften siehe etwa Marx 2010. 
gangs ausmacht. Denn würden wir den Verweis auf die Einstellungen des Akteurs verlieren, wäre auch der Zugriff auf seine Handlungsgründe verspielt. So liefert z.B. eine Erklärung, die das Ankreuzen eines gewissen Kästchens auf dem Wahlzettel ausschließlich aufgrund neuronaler und motorischer Prozesse erklärt, zwar eine Kausalerklärung für die Stimmabgabe eines Akteurs; sie erlaubt allerdings keinerlei Einblicke in die Gründe, weswegen der Akteur dieses Kästchen angekreuzt hat, und die uns seine Handlung aufgrund seiner Überzeugungen und Präferenzen erst verstehen lassen. Dieses Verständnis liefert nur eine Erklärung, die auf die Einstellungen des Akteurs Bezug nimmt. Will Politische Psychologie diesen zentralen Aspekt des Verstehens nicht einbüßen, kann sie folglich durch das Rationalitätsprinzip strukturierte intentionale Sprache nicht außer Acht lassen.

Abschließend lässt sich damit festhalten, dass auch die Politische Psychologie die Rationalität von Akteuren nicht grundsätzlich in Frage stellen kann. Solange sie auf die Ziele und Überzeugungen von Akteuren rekurrieren will, setzt sie einen minimalen Standard von Rationalität voraus. Daher stellt sich auch für Politische Psychologen nicht die Frage, ob Akteure überhaupt rational sind, sondern lediglich, wie rational sie sind. Heißt das in Bezug auf das Konkurrenzverhältnis zwischen Politischer Psychologie und Rational Choice, dass die Kriterien des Umfangs $(C)$ und der Exklusivität $(D)$ verletzt sind? Nicht unbedingt. Denn obwohl Politische Psychologie Grundannahmen über minimale Rationalität mit Rational Choice-Theorien teilen muss, ist damit noch nicht gesagt, dass sie mit allen Rational Choice-Varianten kompatibel ist oder sich die beiden Ansätze ineinander integrieren lassen. Im nächsten Abschnitt werden wir uns diesen Fragen zuwenden.

\section{Der psychologische Kern von Rational Choice}

Nicht nur die Politische Psychologie umschließt verschiedene Forschungsfragen; auch der Begriff „Rational Choice“ wird vielfältig verwendet. Wie bereits erläutert, werden wir uns hier nur auf eine empirisch-analytische Interpretation von Rational Choice beschränken, der zufolge Rational Choice politikwissenschaftliche Phänomene durch Rekurs auf das Handeln rationaler Akteure kausal zu erklären sucht. Alle Rational Choice-Theorien teilen im Kern vier Annahmen: (a) Sie sind dem methodologischen Individualismus verpflichtet, (b) Restriktionen sind eine Bedingung menschlichen Handelns, (c) Handeln ist als Verfolgung von Präferenzen zu verstehen, und (d) Handlungsoptionen werden nutzenmaximierend gewählt. Damit nutzenmaximierendes Verhalten möglich ist, müssen Präferenzen und die mentale Repräsentation der Restriktionen in Form von Überzeugungen gewisse Bedingungen erfüllen: Während Präferenzen u.a. konsistent geordnet werden können müssen, müssen Überzeugungen dergestalt sein, dass sich die Wahrscheinlichkeiten des Eintretens einander ausschließender Ereignisse auf 100 Prozent aufaddieren.

Gleichwohl finden sich unter dem Dach empirisch-analytisch orientierter Rational Choice-Theorien unterschiedliche Strömungen, die sich substantiell in der Verwendungsweise und Deutung der genannten Kernannahmen unterscheiden. Für den Vergleich mit dem Erklärungsprogramm der Politischen Psychologie wer- 
den wir daher auf Seiten der ökonomischen Theorien zwischen realistischen und instrumentalistischen Varianten unterscheiden und diese hier getrennt betrachten.

\subsection{Realistische Rational Choice-Theorie}

Der Forschungsgegenstand von realistischer Rational Choice lässt sich, wie der der Politischen Psychologie, ebenfalls in einen Makro- und einen Mikrobereich unterteilen. Auf der Makroebene geht es um die Erklärung politikwissenschaftlich interessanter Makrophänomene. Dies geschieht wiederum unter Rekurs auf die Mikroebene, und damit auf individuelle Handlungen, die aufgrund der Einstellungen und Überzeugungen von Akteuren vor dem Hintergrund rationaler Entscheidungsprozesse zu erklären sind. Hierbei intendieren realistische Rational Choice-Theorien, die tatsächliche, interne Entscheidungslogik eines Akteurs abzubilden. Anders ausgedrückt: Realistische Rational Choice-Theorie wird als eine Mikrotheorie des Akteurverhaltens verstanden, die das Handeln des Akteurs deskriptiv zutreffend zu beschreiben und erklären versucht. Damit ergeben sich folgende Merkmale für realistische Rational Choice-Theorien:

(a) Deskriptiver Anspruch auf empirische Adäquatheit. Auch realistische Rational Choice-Theoretiker erheben den Anspruch, eine empirisch adäquate Deskription der Handlungsmotivationen eines Akteurs, seiner Situationswahrnehmung sowie seines Entscheidungsverhaltens zu entwickeln. Aus Perspektive realistischer Rational Choice-Theorien zielt eine sozialwissenschaftliche Erklärung sozialer Phänomene somit auf die Identifikation der tatsächlichen Handlungsursachen der Akteure ab (Kunz 2004, S. 48f.).

(b) Einstellungen. Dementsprechend müssen die von dem Akteur in der Handlungssituation wahrgenommenen Handlungsalternativen und ihre subjektive Bewertung abgebildet werden. Dies erfordert, die Situationswahrnehmung der Akteure in Form von Überzeugungen und ihre Zielvorstellungen zu erheben, sowie kausale Hypothesen darüber zu formulieren, wie strukturelle Bedingungen der Handlungssituation Überzeugungen und Präferenzen beeinflussen (Opp 2013a, S. 102f.).

(c) Handlungsselektion. Vor dem Hintergrund der erhobenen Einstellungen muss auch das Entscheidungsverhalten der Akteure theoretisch gefasst werden. Hier halten realistische Rational Choice-Theorien an der Hypothese fest, dass Entscheidungen nutzenmaximierend getroffen werden: Wie in Prinzip $R$ dargelegt, wählen Akteure diejenige Handlungsoption, die den höchsten Erwartungsnutzen verspricht. Dies darf nicht verwechselt werden mit der Behauptung, dass Akteure tatsächlich in ihrem Entscheidungsprozess SEUKalkulationen aufgrund von Nutzenfunktionen durchführen. Es geht vielmehr um die Hypothese, dass Akteure einen kognitiven, auf Präferenzen und Überzeugungen basierten Entscheidungsprozess durchlaufen, der mathematisch aufgrund von Nutzenfunktionen repräsentiert werden kann (Lehtinen u. Kuorikoski 2007, S. 123). Wie diese mathematische Repräsentation genau auszusehen hat - ob Erwartungswerte beispielsweise multiplikativ oder additiv zusammengesetzt sind - ist eine davon unabhängige Frage. 
(d) Stopp-Bedingungen. Eine Handlungserklärung ist somit aus realistischer Rational Choice-Perspektive gelungen, wenn eine empirisch adäquate Bestimmung des Handlungsgrunds eines Akteurs vorliegt und dessen Entscheidungsverhalten mithilfe der Nutzenmaximierungshypothese erklärt werden kann, was wiederum Kenntnis der subjektiven Präferenzen und wahrgenommen Restriktionen voraussetzt. Allerdings stellt realistische Rational Choice auch klare Stopp-Bedingungen an Erklärungen. Erstens ist die Entstehung der Einstellungen der Akteure nicht Forschungsgegenstand von Rational Choice: Obwohl Rational Choice auf kausale Hypothesen zum Einfluss struktureller Bedingungen der Handlungssituation auf Überzeugungen und Präferenzen rekurriert, so ist dennoch nicht ihr Ziel, diesen Einfluss zu erklären. Zweitens ist mit dem Verweis auf Einstellungen die tiefste Ebene der Erklärung erreicht; Rekurs auf neuronale oder genetische Mikrofundierungen von Einstellungen ist in realistischen Rational Choice-Theorien nicht vorgesehen. Folglich bleiben Rational Choice-Erklärungen der intentionalen Sprache verhaftet und die Black Box neuronaler Prozesse geschlossen. Damit einher geht drittens die Beschränkung auf Erklärungen von Verhalten, welches als intentionales Handeln fassbar ist. Verhalten im weiteren Sinne, welches unbewusst abläuft oder generell nicht auf Einstellungen zurückführbar ist, wird nicht beachtet. Nicht zielgerichtetes Verhalten - man denke an wertrationales, affektives oder traditionales Handeln - wird von Rational Choice nur dann betrachtet, wenn es in den theoretischen Rahmen von Rational Choice integriert werden kann, wie zum Beispiel durch Modifikationen von Präferenzordnungen oder durch metatheoretische Betrachtungen zu Entscheidungsmodi vor dem Hintergrund gegebener Situationswahrnehmungen (siehe etwa Esser 1996; Kroneberg 2007).

\subsection{Realistische Rational Choice-Theorie als eine Variante der Politischen Psychologie}

Diese Ausführungen deuten bereits darauf hin, dass realistische Rational ChoiceTheorien ein Teil der Politischen Psychologie sind. Da realistische Rational ChoiceTheorien den Anspruch erheben, Entscheidungsprozesse akkurat zu beschreiben, müssen sie als psychologische Verhaltenstheorien über den kognitiven Entscheidungsprozess eines Akteurs verstanden werden. Allerdings finden sich auch signifikante Unterschiede zwischen beiden Ansätzen, von denen wir hier auf zwei hinweisen möchten. Diese Unterschiede sprechen jedoch nicht gegen die Einordnung der realistischen Rational Choice in die Politische Psychologie.

Erstens fällt auf, dass realistische Rational Choice-Theorien im Gegensatz zur Politischen Psychologie das Gesetz der Nutzenmaximierung nicht antasten. Dies ist zunächst verwunderlich. Denn wenn Vertreter der realistischen Rational ChoiceTheorie der empirischen Erhebung von Einstellungen schon offen gegenüber stehen, weswegen bleiben sie dann gegenüber empirischen Studien zu Entscheidungsmechanismen dennoch verschlossen? Die Aufdeckung diverser systematischer Abweichungen von Rationalitätsstandards - genannt seien hier lediglich Simons (Simon 1957, 
1985, 1986) Überlegungen zur bounded rationality wie auch Studien zu Reflection-, Endowment-, Certainty- oder Sunk-Cost-Effekten (Kahneman et al. 1991) stellt schließlich ernsthafte Einwände gegen den Anspruch der realistischen Rational Choice-Theorie dar, eine psychologisch fundierte Theorie des Entscheidungsprozesses zu präsentieren. Sicherlich reagiert realistische Rational Choice-Theorie auf diese Befunde, indem sie beispielsweise Framing-Effekte bei der Bildung von Überzeugungen einschließt oder durch aufwendige Anreicherungen der Brückenhypothesen, welche strukturelle Faktoren und Akteurswahrnehmung verbinden, diesen Anomalien Herr zu werden versucht (Lindenberg 1993). ${ }^{7}$ Dennoch scheint sie um das Nutzenmaximierungsprinzip einen starken Schutzmantel zu legen (Opp 2013b; Marx 2011). Warum? Zwei Erklärungen wären denkbar. Zum einen könnte argumentiert werden, dass es realistische Rational Choice-Theorien zum Ziel haben, nur denjenigen Bereich von Handeln abzudecken, in dem das Nutzenprinzip tatsächlich empirisch adäquat ist. In diesem Fall wäre das Anwendungsgebiet realistischer Rational Choice ein kleineres Teilgebiet der Politischen Psychologie. Zum anderen könnte das Festhalten am Nutzenmaximierungsprinzip auf gewisse Anforderungen zurückzuführen sein, die realistische Rational Choice-Theoretiker an Erklärungen stellen. Zentral in diesem Kontext sind Überlegungen zur Verallgemeinerbarkeit und zu Gesetzmäßigkeiten, die das Fundament einer jeder Erklärung bilden müssen. Wir werden auf diese wissenschaftstheoretischen Betrachtungen zu Erklärungen im Schlusskapitel unseres Beitrages zurückkommen.

Auch der zweite Unterschied zwischen realistischer Rational Choice und Politischer Psychologie ist in diesem wissenschaftstheoretischen Zusammenhang zu verorten. Dieser bezieht sich auf die genannten Stopp-Bedingungen, die auf der Ebene der Einstellungen zum Halt kommen. Erneut stellt sich die Frage, weswegen für realistische Rational Choice-Theoretiker eine adäquate Erklärung eines Phänomens bereits dann gegeben ist, wenn kausal relevante Einstellungen identifiziert wurden, ohne aber die Entstehung dieser Einstellungen betrachtet zu haben. Wieder ist die Antwort auf diese Frage in den unterschiedlichen Gütekriterien zu finden, die realistische Rational Choice-Theoretiker und Politische Psychologen an Erklärungen stellen und die am Ende dieses Beitrags diskutiert werden.

Hier lässt sich folgendes Zwischenfazit ziehen: Realistische Rational ChoiceTheorie ist ein Spezialfall der Politischen Psychologie, da auch sie den Anspruch erhebt, eine empirisch adäquate, psychologische Mikrotheorie des jeweiligen Explanandums zu liefern. Demnach sind die Konkurrenzkriterien des Umfangs $(C)$ und der Exklusivität $(D)$ verletzt: Da Politische Psychologie mit der realistischen Interpretation des Rational Choice-Ansatzes kompatibel ist, kann keine umfassende Konkurrenz zwischen Politischer Psychologie und Rational Choice per se bestehen. Da sich realistische Rational Choice-Erklärungen als Spezialfälle politisch-psychologischer Erklärungen verstehen lassen, ist auch das Kriterium der Exklusivität nicht erfüllt. Realistische Rational Choice und Politische Psychologie stehen in keinem kategorischen Konkurrenzverhältnis.

7 Hier besteht eine mögliche Reaktion auf diese Anomalien darin, sie umzuinterpretieren: Deutet man sie als Verstöße gegen die Rationalitätsansprüche an Belief-Sets, verstoßen sie ggf. nicht mehr gegen das Nutzenmaximierungsaxiom (Kunz 2004, S. 152). 


\subsection{Instrumentalistische Rational Choice-Theorie}

Die Überlappung zwischen realistischer Rational Choice-Theorie und Politischer Psychologie ist recht offensichtlich. Viel weniger klar ist jedoch, in welchem Verhältnis Politische Psychologie zu instrumentalistisch gedeuteten Rational ChoiceTheorien steht.

Instrumentalistische Theoriemodelle von Rational Choice sind wie die Politische Psychologie und realistische Rational Choice-Theorien an der Erklärung sozialer Makrophänomene interessiert und dem methodologischen Individualismus verpflichtet. Allerdings teilen sie nicht den Anspruch einer ausgearbeiteten psychologischen Mikrotheorie, die Makrophänomene aufgrund empirisch adäquater Erklärung individuellen Handelns fassen möchte, sondern streben lediglich eine sogenannte Mikrofundierung ihrer Makroerklärungen an. Die folgenden Punkte fassen die wichtigsten Charakteristika instrumentalistischer Rational ChoiceTheorie zusammen:

(a) Kein deskriptiver Anspruch auf empirische Adäquatheit. Instrumentalistische Rational Choice-Theoretiker zielen nicht auf empirisch adäquate Beschreibungen des tatsächlich ablaufenden psychologischen Entscheidungsprozesses ab. Stattdessen begreift diese Variante von Rational Choice den Verweis auf Einstellungen als ein Instrument, das sich zur Erklärung und Prognose sozialer Phänomene eignet, ohne die Black Box individueller Entscheidungsfindungen öffnen zu wollen. Daher sprechen manche Vertreter des Instrumentalismus auch davon, dass sich Akteure unter bestimmten Bedingungen so verhalten, ,als ob` sie rational kalkulieren oder gewissen Präferenzen folgen würden. Die zentrale These des Instrumentalismus ist dementsprechend nicht, dass Akteure tatsächlich gewisse Einstellungen haben und kognitive Entscheidungsprozesse durchlaufen, sondern dass ihr Verhalten konsistent ist mit Interpretationen entlang solcher ,als ob' Zuschreibungen (Satz u. Ferejohn 1994).

(b) Einstellungen und Handlungsselektion. Dementsprechend werden Einstellungen nicht empirisch erhoben, sondern innerhalb theoretischer Modelle im Einklang mit Rationalitätsstandards postuliert. Der theoretische Status von Präferenzen ist hierbei im Rahmen instrumentalistischer ökonomischer Theorien umstritten. Manche lehnen die Existenz mentaler Zustände ab und sehen Präferenzen und Überzeugungen lediglich als theoretische Konstrukte, die uns ermöglichen, beobachtbares Verhalten zu beschreiben. ${ }^{8}$ Andere zweifeln die Existenz von Einstellungen nicht an, beharren aber darauf, dass diesen Einstellungen keinerlei kausale Rolle innerhalb von Rational Choice-Erklärungen zugeschrieben werden darf, da Einstellungszuschreibungen als fiktive Annahmen verstanden werden müssen. Gleiches gilt für die Unterstellung, dass Akteure Nutzen maximieren (Lovett 2006, S. 256-259). Da nicht behauptet wird, dass Akteure tatsächlich Nutzenmaximierer sind oder tatsächlich vollkommen konsistente Präferenzen besitzen, können Experimente,

8 Ein Grund hierfür liegt in Zweifeln über die Messbarkeit von Einstellungen. Wir blenden diese Zweifel hier aus. 
die diese Annahmen durch das Aufdecken systematischer Anomalien zu widerlegen suchen, als irrelevant eingestuft und ignoriert werden.

(c) Strukturen. Da Annahmen über Entscheidungsprozesse folglich als instrumentelle ,als ob` Aussagen verstanden werden, tragen sie keinerlei Erklärungslast. Diese Last wird vielmehr auf strukturelle Faktoren, d.h. situative Restriktionen verlagert. Die Analyse dieser situativen Bedingungskonstellationen, die das Handeln der Akteure extern strukturieren, soll die Problematik der Erfassung individueller Präferenzstrukturen und das Betreiben politischer Psychologie vermeiden. In diesem Zusammenhang ist auch die weitverbreitete Beschränkung des Anwendungsgebiets instrumentalistischer ökonomischer Theorien auf Situationstypen zu verstehen, in denen Verhaltensänderungen vollständig auf Restriktionsänderungen zurückgeführt werden sollen und in denen strukturelle Anreize Wahl der zur Verfügung stehenden Handlungsalternativen stark einschränken. Zu nennen wären hier beispielsweise Hochkostensituationen (Mensch 1999, 2000) oder institutionelle Kontexte mit starkem Wettbewerbsdruck, die langfristig nicht-rationale Akteure aus dem Spiel nehmen oder instrumentelle Güter in den Vordergrund rücken, deren Nützlichkeit für alle Akteure unterstellt werden kann (Zintl 2001). Daher könne Instrumentalisten zufolge in solchen Situationen von einem strukturellen Determinismus ausgegangen werden, der psychologische Betrachtungen überflüssig macht: Instrumentalisten „concentrate on the logic of the agent's situation and are spared the complexities of the psychology of the agent in that situation" (Latsis 1972, S. 211).

(d) Stopp-Bedingungen. Mit diesem Fokus auf strukturelle Faktoren und ,als ob Mikrofundierungen geht die Stopp-Bedingung einher, Erklärungen in strukturellen Faktoren und postulierten Einstellungen zu verankern. Da der Entscheidungsprozess von Akteuren fiktiv gedeutet wird, verbieten sich vor diesem Hintergrund sowohl Analysen zur Einstellungsentstehung als auch der Rekurs auf die neuronale Basis von Einstellungen.

\subsection{Die Notwendigkeit von Psychologie in instrumentalistischen Rational Choice- Theorien}

Da die Erklärungslast aus Sicht instrumentalistischer ökonomischer Theorien auf situativen Faktoren liegt, wohingegen Entscheidungsprozesse lediglich als Fiktion postuliert werden, scheinen instrumentalistische Rational Choice-Theorien und Politische Psychologie grundverschieden zu sein: Wohingegen erstere trotz Mikrofundierung strukturalistische Theorien sind, sind letztere zutiefst in der Psychologie verhaftet. Gegen diesen Eindruck möchten wir nun argumentieren: Selbst die instrumentalistische Variante erklärender Rational Choice-Theorie muss psychologischen Faktoren letztlich eine kausale Rolle zuschreiben und kann nicht bei Als-ob-Annahmen stehen bleiben.

Der Grund hierfür wird am leichtesten ersichtlich, wenn man die folgenden beiden Szenarien betrachtet. Im ersten Szenario haben wir es mit einer starken Wettbewerbssituation zu tun, wie z.B. dem politischen Wettbewerb der Parteien um 
Stimmen. Im zweiten Szenario liegt keine solche Wettbewerbssituation vor; betrachten wir hier z.B. das Verhalten von Wählern. Es wird nun oft festgestellt, dass Rational Choice im ersten Szenario erfolgreich ist, im zweiten Beispiel allerdings nicht überzeugen kann. Verantwortlich für diesen Unterschied werden wiederum Strukturen gemacht: Wie Satz und Ferejohn (1994, S. 72) zusammenfassen:

(...) rational-choice explanations are most plausible in settings in which individual action is severely constrained, and thus where the theory gets its explanatory power from structure-generated interests and not from actual individual psychology. In the absence of strong environmental constraints, we believe that rational choice is a weak theory, with limited predictive power.

The primary reason for the asymmetry of explanatory success is that (...) voters face less competitive environments than (...) parties. We fully realize the irony of our contention: the theory of rational choice is most powerful in contexts where choice is limited.

Doch wie genau entfalten Strukturen ihre Kausalwirkung in den beiden Szenarien? Es wäre unplausibel anzunehmen, dass Strukturen manchmal kausal wirksam sind und manchmal eben nicht. Wie Satz und Ferejohn (1994) betonen, ist der Unterschied in den beiden Fällen vielmehr darin zu verorten, dass im ersten Szenario starke Strukturen spezifische Interessen der Parteien implizieren, wohingegen schwache Strukturen im Beispiel der Wähler dies nicht erlauben. Gleichzeitig werden die den Parteien so zugeschriebenen Interessen vor dem instrumentalistischen Hintergrund natürlich auch im ersten Szenario lediglich aufgrund der Position der Parteien in sozialen Strukturen fiktiv unterstellt, und nicht auf der Basis individueller psychologischer Faktoren zugeschrieben. Doch wieder stellt sich die Frage: Weswegen führen die ,als $\mathrm{ob}^{`}$ Zuschreibungen von Präferenzen im Fall starker Strukturen zu erfolgreichen Rational Choice-Erklärungen, im Fall schwacher Strukturen jedoch nicht, wo alle Einstellungszuschreibungen doch angeblich lediglich fiktiven Charakter haben? Unserer Meinung nach finden wir nur dann eine plausible Antwort auf den Unterschied zwischen den beiden Szenarien, wenn der fiktive Status von Einstellungszuschreibungen aufgegeben wird. Das heißt: Rational Choice greift bei starken Strukturen, wie sie etwa für Hochkostensituationen kennzeichnend sind, besser, weil starke Strukturen die Zuschreibung tatsächlicher Präferenzen und Situationswahrnehmungen erleichtern. Gleichzeitig ist Rational Choice im Fall schwacher Strukturen weniger erfolgreich, weil schwache Strukturen keine klaren Hypothesen über tatsächliche Präferenzen und Situationswahrnehmungen nahelegen. In anderen Worten: Strukturen sind in beiden Szenarien kausal wirksam. Die Fokussierung auf starke Strukturen entspricht allerdings einer Selektion jener Anwendungsfälle, in denen empirische Hypothesen über Präferenzen leichter zu formulieren sind, da Strukturen Präferenzen stärker begrenzen. Dies sollte uns jedoch nicht zu dem Schluss verleiten, dass Präferenzen deswegen lediglich instrumentell zu postulieren sind. Folglich darf der Erfolg von Rational Choice in starken strukturalistischen Kontexten nicht ausschließlich in Strukturen verortet werden, wohingegen Präferenzen eine kausale Rolle abge- 
sprochen wird, sondern liegt in der Kombination von kausal wirksamen Strukturen und kausal wirksamen Präferenzen. ${ }^{9}$

Um Missverständnisse zu vermeiden, sei klargestellt, dass wir Rational ChoiceModellen, die mit ,als ob'-Hypothesen operieren und keinerlei Anspruch auf empirische Adäquatheit erheben, in gewissen Kontexten - wie z.B. im Rahmen explikativer oder normativer Forschungsinteressen - keineswegs ihre Daseinsberechtigung absprechen möchten. Diese Daseinsberechtigung bezieht sich aber auf Anwendungsbereiche, die eben nicht Erklärungen anstreben, sondern andere Ziele verfolgen. Insofern Rational Choice-Theorien eine erklärende Funktion übernehmen sollen, sind instrumentalistische Deutungen fehlgeleitet, da selbst strukturalistische Rational Choice-Theorien psychologisch verstanden werden müssen: Selbst wenn Strukturen gewisse Einstellungszuschreibungen plausibel machen, sind diese als empirische Hypothesen über kausal wirksame psychologische Faktoren zu interpretieren. Die Konkurrenzkriterien $(C)$ und $(D)$ sind demnach erneut verletzt: Die instrumentalistische Version der Rational Choice-Theorie geht in der realistischen Variante auf und ist damit nicht nur mit Politischer Psychologie kompatibel, sondern ist in diese integrierbar.

\section{Sozialwissenschaftliche Erklärungen - rational und/oder psychologisch?}

Unser Ergebnis mag den Schein erwecken, dass alle Mauern zwischen Politischer Psychologie und Rational Choice eingerissen sind: Wir haben argumentiert, dass Politische Psychologie von minimaler Rationalität ausgehen muss, wohingegen Rational Choice die kausale Rolle psychischer Faktoren nicht anzweifeln kann. Eine kategorische Konkurrenz zwischen beiden Ansätzen besteht demnach nicht. Impliziert dieses Ergebnis, dass keine relevanten Unterschiede zwischen diesen Programmen bestehen? Bedeutet es anders ausgedrückt, dass nichts auf dem Spiel steht, wenn wir uns zwischen einem politisch-psychologisch orientierten oder einem rationalitätsbasierten Forschungsdesign entscheiden sollen? Beide Fragen sind unserer Meinung nach zu verneinen. Denn selbst wenn kategorische Konkurrenz zurückgewiesen werden muss, bestehen gewisse Unterschiede zwischen diesen, vor allem im Hinblick auf Stopp-Bedingungen und Verweise auf die individuelle Vielfalt der Informationsverarbeitung und Handlungsselektion. Obwohl es aus unserer Sicht also verfehlt wäre, zu fragen, ob wir entweder Politische Psychologie oder Rational Choice akzeptieren sollten, stellt sich nach wie vor die Frage, ob sich sozialwissenschaftliche Erklärungen an politisch-psychologischen Ansätzen oder an rationalitätsbasierten Erklärungsmustern orientieren sollten.

9 Hier könnte eingewandt werden, dass dieses Argument es versäumt, zwei verschiedene Deutungen von Rational Choice-Erklärungen klar voneinander zu trennen, nämlich eine interne, die die Binnenperspektive des Akteurs einnimmt, und eine evolutionäre, die eine dem Akteur externe Perspektive sucht (siehe Kliemts (1996) Diskussion eines solchen Kritikpunkts). Unserer Meinung nach geht dieser Einwand jedoch fehl, da bei der evolutionären Interpretation intentionales Handeln eben nicht Teil des Kausalmechanismus ist. Wie Kliemt (1996, S. 89) es auf den Punkt bringt: „Insoweit kann man nur feststellen, daß die [evolutionär gedeuteten] Rational Choice-Ansätze mit den wirklichen Kausalmechanismen, die die Entwicklung sozialer Prozesse real beeinflussen, nichts zu tun haben." Ein solcher evolutionärer Ansatz mag eine Erklärung für ein Explanandum liefern; diese Erklärung sollte jedoch nicht als Rational Choice-Erklärung verstanden werden. 
Anders ausgedrückt: Wie psychologisch bzw. Rational Choice-fundiert sollte eine Erklärung sozialen Handelns ausfallen?

Um einen klaren Blick auf diese Frage zu gewinnen, ist zu überlegen, welche Erklärungstiefe bei gleichem Forschungsinteresse erreicht werden sollte. Zur Verdeutlichung dieser Überlegung ist es hilfreich, die folgenden drei, zugegebenermaßen stilisierten, Erklärungen desselben Makrophänomens $X$ zu vergleichen:

(PSYCHOLOGIE) Makrophänomen $X$ ist dann zufriedenstellend erklärt, wenn die kausale Rolle von Strukturen, von empirisch erhobenen Einstellungen und Entscheidungsprozessen sowie deren Entstehungsprozess bzw. neuronale und/oder genetische Basis identifiziert wurde.

$\left(\mathrm{RC}_{\mathrm{R}}\right) \quad$ Makrophänomen $X$ ist dann zufriedenstellend erklärt, wenn die kausale Rolle von Strukturen und deren Wirkung auf empirisch erhobene Einstellungen vor dem Hintergrund der Nutzenmaximierung identifiziert wurde.

$\left(\mathrm{RC}_{\mathrm{I}}\right) \quad$ Makrophänomen $X$ ist dann zufriedenstellend erklärt, wenn die kausale Rolle von Strukturen und daraus abgeleiteten Einstellungen vor dem Hintergrund der Nutzenmaximierung identifiziert wurde.

Welches Erklärungsmuster sollten wir akzeptieren? Ist eine Entscheidung besser, wenn sie Einstellungen als endogene Faktoren versteht? Ist sie besser, wenn Einstellungen empirisch erhoben werden? Kurzum: Wie tief muss eine Erklärung sein?

Es lohnt an dieser Stelle, an drei substantielle Gütekriterien, die an Kausalerklärungen gerichtet werden, zu erinnern. Erstens müssen Erklärungen mit Hempel und Oppenheim (1948, S. 137) empirisch adäquat sein: Die Annahmen, die eine Erklärung trifft, müssen wahr sein. Zweitens ist das Ziel, Kausalprozesse zu identifizieren, erst dann erreicht, wenn strukturell beeinflusste intentionale Handlungen miteinbezogen werden (Little 1995, S. 35). Drittens müssen Erklärungen auf Gesetze rekurrieren: Sie müssen allgemeine Sätze über den Zusammenhang verschiedener Variablen enthalten. Eine Erklärung ist demnach dann eine gute Erklärung, wenn sie die tatsächlichen Ursachen des jeweiligen Makrophänomens mit Bezug auf eine Mikrotheorie identifiziert und in allgemeine Gesetzmäßigkeiten einbettet. In Anbetracht dieser substantiellen Gütekriterien schlagen wir nun folgende, zugegebenermaßen tentative Antwort auf die Frage nach der Tiefe von Erklärungen vor:

Die Forderung nach der empirisch adäquaten, mikrotheoretischen Identifikation kausaler Prozesse impliziert, dass Einstellungen sowie die Brückenhypothesen, die diese Einstellungen mit strukturellen Faktoren verbinden, empirisch erhoben und getestet werden müssen. Dieses Kriterium schließt daher $\left(\mathrm{RC}_{\mathrm{I}}\right)$ aus: Wird $\left(\mathrm{RC}_{\mathrm{I}}\right)$ rein instrumentalistisch interpretiert, ist es wie oben erläutert als Rational Choice-Erklärung abzulehnen, da fiktiven Einstellungen eben keine kausale Wirksamkeit zugesprochen wird. Nimmt man hingegen eine psychologische Lesart von $\left(\mathrm{RC}_{\mathrm{I}}\right)$ an, die Einstellungen zwar eine kausale Rolle zuspricht, Präferenzen aber lediglich aufgrund von Plausibilitätsüberlegungen aus Strukturen theoretisch ab- 
leitet, kann $\left(\mathrm{RC}_{\mathrm{I}}\right)$ höchstens als potentielle Erklärung eines Phänomens betrachtet werden, deren Überprüfung auf empirische Adäquatheit allerdings noch aussteht. $\mathrm{Da}$ diese Überprüfung empirische Untersuchungen von Einstellungen und Brückenhypothesen impliziert, fällt eine gute $\left(\mathrm{RC}_{\mathrm{I}}\right)$-Erklärung daher in eins mit $\left(\mathrm{RC}_{\mathrm{R}}\right)$. Anders ausgedrückt: Um von der empirischen Adäquatheit einer Erklärung überzeugt zu sein, können wir nie bei $\left(\mathrm{RC}_{\mathrm{I}}\right)$ stehen bleiben, sondern müssen immer den weiteren Schritt zu $\left(\mathrm{RC}_{\mathrm{R}}\right)$ gehen. Dieses Ergebnis ist zudem unabhängig von Anwendungskontexten. Egal, ob wir beispielsweise an Hoch- oder an Niedrigkostensituationen interessiert sind, muss die empirische Adäquatheit unterstellter Einstellungen und Brückenhypothesen nachgewiesen werden.

Mit dem Wegfall von $\left(\mathrm{RC}_{\mathrm{I}}\right)$ bleiben daher nur $\left(\mathrm{RC}_{\mathrm{R}}\right)$ und (PSYCHOLOGIE) als Erklärungsmuster übrig. Hier könnte vorgeschlagen werden, dass das Gütekriterium der empirischen Adäquatheit auch in diesem Fall eine Entscheidung zwischen $\left(\mathrm{RC}_{\mathrm{R}}\right)$ und (PSYCHOLOGIE) herbeiführt, und zwar zugunsten von (PSYCHOLOGIE). Denn wenn wir schon an Kausalprozessen interessiert sind, so das Argument, dann sollten wir auch auf die tiefste erreichbare Ebene dieser Prozesse vordringen und gegebenenfalls neurologische und genetische Faktoren einbeziehen. Wir teilen diese Argumentation nur zum Teil. Sicherlich schadet es einer Erklärung eines Makrophänomens nicht, eine neurologische Mikrofundierung von Entscheidungsprozessen anzubieten. Auch ist unbestritten, dass das Verfolgen bestimmter individualpsychologischer Fragestellungen, wie z.B. von Persönlichkeitsentwicklungen, den Rekurs auf genetische oder neurologische Erkenntnisse verlangt und dass solche Erkenntnisse Eingang in unsere Mikrotheorien finden sollten. Allerdings haben wir Zweifel daran, dass die Erklärung von sozialen $\mathrm{Ma}$ krophänomenen grundsätzlich durch einen solchen Rekurs bereichert wird. Wie oben angedeutet ist eine sozialwissenschaftliche Erklärung erst dann wertvoll, wenn sie in einer Sprache verfasst ist, die uns Handeln verstehen lässt. Dies wird geleistet von intentionaler Sprache, die auf die Präferenzen und Überzeugungen von Akteuren Bezug nimmt. Daher müssen genetische und neurologische Erkenntnisse an propositionale Einstellungen gebunden werden, welche die Basis einer sozialwissenschaftlichen Erklärung bilden. (PSYCHOLOGIE) ist damit $\left(\mathrm{RC}_{\mathrm{R}}\right)$ nicht zwangsläufig überlegen.

Das dritte Gütekriterium hingegen - Erklärungen müssen auf Gesetze rekurrieren und verallgemeinerbar sein - unterstützt unseres Erachtens allerdings für $\left(\mathrm{RC}_{\mathrm{R}}\right)$ und spricht für (PSYCHOLOGIE) zumindest ein gewisses Wort der Warnung aus. Ein solches allgemeines Gesetz sozialwissenschaftlicher Erklärungen ist unserer Meinung nach im Einklang mit Rational Choice-basierten Theorien im Rationalitätsprinzip $R$ zu verorten. Erstens eignet sich dieses Prinzip als allgemeines Gesetz, da es einen eindeutigen Zusammenhang zwischen Einstellungen und Handlungen herstellt. Zweitens ist dieses Gesetz wie oben erläutert unerlässlich, wenn wir über menschliche Aktivität als Handlungen sprechen wollen. Das Rationalitätsprinzip kann damit als allgemeiner theoretischer Kern des sozialwissenschaftlichen Forschungsprogramms verstanden werden. Dies verschafft $\left(\mathrm{RC}_{\mathrm{R}}\right)$ Erklärungen einen nicht zu vernachlässigenden Vorteil, da diese explizit am Nutzenmaximierungsprinzip festhalten. Wir haben bereits oben erläutert, dass auch (PSYCHOLOGIE) das Rationalitätsprinzip nicht grundsätzlich ablehnen 
kann, wodurch (PSYCHOLOGIE) und $\left(\mathrm{RC}_{\mathrm{R}}\right)$ näher aneinander rücken, als ursprünglich gedacht. Würde sich (PSYCHOLOGIE) in Auseinandersetzung mit nachgewiesenen Anomalien jedoch zu einer idiosynkratischen Individualwissenschaft entwickeln, die Verallgemeinerung und universale Gesetze unmöglich machte, würde sie vor dem Hintergrund des dritten Gütekriteriums in ernste Schwierigkeiten geraten. Dementsprechend bietet sich folgende Interpretation des Verhältnisses von Rational Choice und Politischer Psychologie an: Da Theoriemodifikationen nicht am Kern des Forschungsprogramms vorzunehmen sind, sondern durch Änderungen des Schutzgürtels geleistet werden müssen (Lakatos 1964, 1974), kann Politische Psychologie im Zusammenhang solcher Modifikationen im Sinne einer psychologischen Anreicherung von Brückenhypothesen verstanden werden, die in Rational Choice-Theorien verwendet werden und sich sowohl auf die Situationswahrnehmung des Akteurs wie auch auf dessen Zielvorstellungen beziehen können.

Eine detailliertere Entwicklung dieser Ideen wäre natürlich erforderlich, um eine überzeugende und vollständige Konzeption sozialwissenschaftlicher Erklärung anzubieten. Vor dem Hintergrund der genannten Gütekriterien an Erklärungen lautet allerdings unsere vorläufige Antwort auf die Frage, ob sich sozialwissenschaftliche Erklärungen eher an politisch-psychologischen Ansätzen oder an rationalitätsbasierten Erklärungsmustern ausrichten sollten: Greife auf psychologische Theorien zur Entwicklung von Brückenhypothesen zurück, doch orientiere dich für die Erklärung von Handlungen am Rationalitätsprinzip, wie es in $\left(R_{R}\right)$ formuliert ist.

\section{Fazit}

,Auf die Couch!‘ lautet die Überschrift dieses Beitrags. Die Wahl dieser Überschrift liegt in unserer Überzeugung begründet, dass das Verhältnis von Politischer Psychologie und Rational Choice von Missverständnissen belastet ist. Weder teilen wir die instrumentalistische Position mancher Rational Choice-Vertreter, dass Handlungserklärungen ohne eine psychologische Mikrotheorie auskommen können und sich auf die Benennung struktureller Faktoren reduzieren lassen, noch halten wir einen Verzicht auf das Rationalitätsprinzip, wie es von manchen Vertretern der Politischen Psychologie proklamiert wird, für durchführbar. Wir haben stattdessen dafür plädiert, dass die Politische Psychologie minimale Rationalität und Rational Choice die kausale Rolle empirisch erhobener, psychischer Faktoren anerkennen muss. Folglich besteht keine kategorische Konkurrenz zwischen beiden Ansätzen. Stattdessen konzentrieren sie sich auf unterschiedliche Elemente einer sozialwissenschaftlichen Handlungserklärung, für deren Gelingen jedoch beides benötigt wird: psychologisch informierte kausale Hypothesen über die Effekte von Strukturen auf Informationsverarbeitung, Präferenzen und kognitive Prozesse, wie auch den Rekurs auf das Rationalitätsprinzip als allgemeines Handlungsgesetz. 


\section{Literatur}

Capara, Gian Vittorio, Claudio Barbaranelli, und Philip Zimbardo. 1999. Personality profiles and political parties. Political Psychology 20 (1): S. 175-197.

Catellani, Patrizia. 2004. Political psychology. Overview. In Encyclopedia of applied psychology. Hrsg. Charles Donald Spielberger, S. 51-65. London: Elsevier.

Davidson, Donald. 1980a. Actions, Reasons and Causes. In ders.: Essays on Actions and Events, S. 3-20. Oxford: Clarendon Press.

Davidson, Donald. 1980b. Mental Events. In ders.: Essays on Actions and Events, S. 207228. Oxford: Clarendon Press.

Davidson, Donald. 1984. Radical Interpretation. In ders.: Inquiries into truth and interpretation, S. 125-139. Oxford: Clarendon Press.

Davidson, Donald. 2004. Problems of Rationality. Oxford: Oxford University Press.

Esser, Hartmut. 1996. Definition der Situation. Kölner Zeitschrift für Soziologie und Sozialpsychologie 48 (1): S. 1-34.

Farnham, Barbara. 1990. Political cognition and decision-making. Political Psychology 11 (1): S. 83-111.

Fazio, Russell H. 1990. Multiple Processes by which Attitudes Guide Behavior: The Mode Model as an Integrative Framework. In Advances in Experimental Social Psychology Bd. 23. Hrsg. Mark P. Zanna, S. 75-109. London: Academic Press.

Green, Donald P., und Ian Shapiro. 1996. Pathologies of rational choice theory. A critique of applications in political science. Cambridge: Cambridge University Press.

Hempel, Carl G. (1961): Rational Action. In Proceedings and Addresses of the American Philosophical Association 35: S. 5-23.

Hempel, Carl G., und Paul Oppenheim. 1948. Studies in the Logic of Explanation. Philosophy of Science 15 (2): S. 135-175.

Hoggett, Paul, und Simon Thompson. 2012. Introduction. In dies.: Politics and the Emotions. The affective turn in contemporary political studies: S. 1-19. New York: Continuum.

Kahneman, Daniel, und Amos Tversky. 1981. The Framing of Decisions and the Psychology of Choice. Science 211 (4481): S. 453-458.

Kahneman, Daniel, Jack L. Knetsch, und Richard H. Thaler. 1991. Anomalies, the Endowment Effect, Loss Aversion and Status Quo Bias. Journal of Economic Perspective 5 (1): S. 193-206.

Kliemt, Hartmut. 1996. Rational Choice-Erklärungen? In Handlungs- und Entscheidungstheorie in der Politikwissenschaft. Eine Einführung in Konzepte und Forschungsstand. Hrsg. Ulrich Druwe und Volker Kunz, S. 83-105. Opladen: Leske und Budrich.

Kroneberg, Clemens. 2007. Wertrationalität und das Modell der Frame-Selektion. Kölner Zeitschrift für Soziologie und Sozialpsychologie 59 (2): S. 215-239.

Kunz, Volker. 2004. Rational Choice. Frankfurt: Campus.

Lakatos, Imre. 1964. Proofs and Refutations. 4. In British Journal for the Philosophy of Science 14 (56): S. 296-342.

Lakatos, Imre. 1974. Falsifikation und Methodologie wissenschaftlicher Forschungsprogramme. In Kritik und Erkenntnisfortschritt. Hrsg. Imre Lakatos und Alan Musgrave, S. 89-189. Braunschweig: Vieweg.

Latsis, Spiro J. 1972. Situational Determinism in Economics. The British Journal for the Philosophy of Science 23 (3): S. 207-245. 
Lehtinen, Aki, und Jaakko Kuorikoski 2007. Unrealistic Assumptions in Rational Choice Theory. Philosophy of the Social Sciences 37: S. 155-138.

Lindenberg, Siegwart. 1993. Framing, Empirical Evidence, and Applications. In Jabrbuch für Neue Politische Ökonomie. Hrsg. Philipp Herder-Dorneich, Karl-Ernst Schenk und Dieter Schmidtchen, S. 11-49. Tübingen: Mohr Siebeck.

Little, Daniel. 1995. Causal Explanation in the Social Sciences. Southern Journal of Philosophy 34 (S1): S. 31-56.

Lodge, Milton, und Charles S. Taber. 2013. The Rationalizing Voter. Cambridge: Cambridge University Press.

Lovett, Frank. 2006. Rational Choice Theory and Explanation. Rationality and Society 18 (2): S. 237-272.

Marcus, George E. 2013. Political psychology. Neuroscience, genetics, and politics. New York: Oxford University Press.

Marx, Johannes. 2010. Rationalität, Hermeneutik und Neurowissenschaften. Eine Auseinandersetzung mit den kultur- und neurowissenschaftlichen Herausforderungen ökonomischer Theorien vor dem Hintergrund der Theorie von Donald Davidson. In Jabrbuch für Handlungs- und Entscheidungstheorie. Bd. VI: Schwerpunkt neuere Entwicklungen des Konzepts der Rationalität und ihre Anwendungen. Hrsg. Joachim Behnke, Thomas Bräuninger und Susumu Shikano, S. 130-161. Wiesbaden: Springer VS.

Marx, Johannes. 2011. Zum Status und zur Leistungsfähigkeit von Annahmen in der Ökonomik. Zeitschrift für Internationale Beziehungen 18 (1): S. 125-132.

Mensch, Kirsten. 1999. Die segmentierte Gültigkeit von Rational-Choice-Erklärungen. Warum Rational-Choice-Modelle die Wablbeteiligung nicht erklären können. Opladen: Leske und Budrich.

Mensch, Kirsten. 2000. Niedrigkostensituationen, Hochkostensituationen und andere Situationstypen. Ihre Auswirkungen auf die Möglichkeiten von Rational-Choice-Erklärungen. Kölner Zeitschrift für Soziologie und Sozialpsychologie 52 (2): S. 246-263.

Monroe, Kristen Renwick, William Chiu, Adam Martin, und Bridgette Portman. 2009. What Is Political Psychology? Perspectives on Politics 7 (4): S. 859-882.

Opp, Karl-Dieter. 2013a. Methodologie der Sozialwissenschaften. Einführung in die Probleme ihrer Theoriebildung und praktische Anwendung. Wiesbaden: Springer VS.

Opp, Karl-Dieter. 2013b. Norms and Rationality. Is moral behavior a form of rational action? Theory and Decision 74 (3): S. 383-409.

Rothermund, Klaus, und Andreas B. Eder. 2011. Allgemeine Psychologie. Motivation und Emotion. Wiesbaden: VS Verlag.

Satz, Debra, und John Ferejohn. 1994. Rational Choice and Social Theory. Journal of Philosophy 91 (2): S. 71-87.

Schoen, Harald. 2006. Der demokratische Musterbürger als Normalfall? Kognitionspsychologische Einblicke in die black box politischer Meinungsbildung. Politische Vierteljabresschrift 47 (1): S. 89-101.

Simon, Herbert A. 1957. Models of Man. New York: John Wiley and Sons.

Simon, Herbert A. 1985. Human Nature in Politics: The Dialogue of Psychology with Political Science. The American Political Science Review 79 (2): S. 293-304.

Simon, Herbert A. 1986. Rationality in Psychology and Economics. The Journal of Business 59 (4): S. 209-224. 
Small, Deborah, Jennifer Lerner, und Baruch Fischhoff. 2006. Emotion priming and attributions for terrorism: Americans' reactions in a national field experiment. Political Psychology 27 (2): S. 289-298.

Tiefensee, Christine. i.E. Why the Realist-Instrumentalist Debate about Rational Choice Rests on a Mistake.

Tversky, Amos. 1975. A Critique of Expected Utility Theory: Descriptive and Normative Considerations. Erkenntnis 9 (2): S. 163-173.

Zintl, Reinhard. 2001. Rational Choice as a Tool in Political Science. Associations 5 (1): S. $35-50$. 